\title{
A New Revisited Compression Technique through Innovative Partiotion Group Binary Compression: A Novel Approach
}

\author{
V. Hari Prasad
}

\begin{abstract}
Day by day the ratio of living organisms are increased and its accumation in the bilogical databases creating a major task. In this connectin some of the classical algorithms are strived into the world and fails to compress genetic sequences due to the captcha of alphabets. Existing substitution techniques will work on repetitive and non repetitiveness of bases of DNA and they achieve Best compression rates if any sequence may contain tandem repeats. But the category of grasses like maize and rice contains very less repeats (nearer of 27) over 67789 total bps. By working existing techniques on such sequences the results are not bountiful and running on worst case comparisons. This paper introduces a novel Innovative partition group Binary compression technique yields first art compression rates which is far better than existing techniques. This algorithm is develpoed based on comparative study of existing algorithms and which is more applicable for non tandem repeats of DNA sequences in genomes.
\end{abstract}

Index Terms-Biocompress, dnabit compress, genbit compress, huffbit compress, encode, decode.

\section{INTRODUCTION}

The human genome was finally deciphered! In other words, scientists have succeeded in reading the chain of more than 3 billion base pairs that constitute the DNA molecule of humans; this process is called, sequencing. That daunting task required new analytical methods created by bioinformatics .Bio informatics is nothing but information technology is applied in terms of biological databases. To maintain such huge databases we require efficient Bio informatics computational tools to store and process data in a more efficient way. Today more and more DNA sequences are storing in biological databases so the size of the databases is growing in an exponential manner. Thus need of compression arises and it is becoming a vital challenge, In this connection many universal algorithms are came into the existence but they fails to compress genetic sequences due to the specificity of "text". Some classical algorithms are also introduced but they are running on negative compression rates. Compression may be in two flavors one is lossless and other is loss. Loss compression can be applicable for multimedia applications like image, audio and video. In multimedia applications if we remove some unused pixels also resultant may not variant like removing noise from audio or removing unnecessary pixels from images But Text

Manuscript received December 12, 2012; revised March 27, 2013.

V. Hari Prasad is with Department of CSE (e-mail: hariprasadvemulapati@gmail.com). compression is always loss less even after decoding the entire encoded text we have to retain its original property. DNA can be encoded in four letter alphabets like text $\{A, C, G$, and $T$ \}.Thus each Base of symbol (Base) can be represented by two bits. General purpose compression algorithms do not perform well with biological sequences. Giancarlo et al. [1], [2] have provided a review of compression algorithms designed for biological sequences. Finding the characteristics and comparing Genomes is a major task (Koonin 1999 [3]; Wooley 1999 [4]). In mathematical point of view, compression implies understanding and comprehension ( $\mathrm{Li}$ and Vitanyi 1998) [5]. Compression is a great tool for Genome comparison and for studying various properties of Genomes. DNA sequences, which encode life should be compressible. It is well known that DNA sequences in higher eukaryotes contain many tandem repeats, and essentials genes (like rRNAs) have many copies. It is also proved that genes duplicate themselves sometimes for evolutionary purposes. All these facts conclude that DNA sequences should be compressible. The compression of DNA sequences is not an easy task. (Grumback and Tahi 1994 [6], Rivals et al. 1995 [7]; Chen et al. 2000 [8]) DNA sequences consists of only four nucleotides bases $\{A, C, G, T\}$. Two bits are enough to store each base. The standard compression software's such as "compress", "gzip", "bzip2", "winzip" expanded the DNA genome file more than compressing it.

Most of the Existing software tools worked well for English text compression (Bell et al. 1990 [9]) but not for DNA Genomes. There are many text compression algorithms available having quite a good compression ratio. But they have not been proved well for compressing DNA sequences as the algorithm does not incorporate the characteristics of DNA sequences even though DNA sequences can be represented in simple text form. DNA sequences are comprised of just four different bases labeled $A, T, C$, and $G$ (for adenine, thymine, cytosine, and guanine respectively). $\mathrm{T}$ pairs with $A$, and $G$ pairs with $C$. Each base can be represented in computer code by a two character binary digit, two bits in other words, $A(00), C(01), G(10)$, and $T(11)$. At first glance, one might imagine that this is the most efficient way to store DNA sequences. Like the binary alphabet $\{0,1\}$ used in computers, the four-letter alphabet of DNA $\{A, T, C$, and $G$ \} can encode messages of arbitrary complexity when encoded into long sequences.

\section{A. Plan of the Paper}

This paper is organized as follows. Section II describes general compression algorithms. Section III describes related existing algorithms to compress genome data. Section VI describes proposed algorithms analysis how it is better one 
than existing techniques. Section V describes comparative study on a sample sequence. Section VI is concluding with future work.

\section{General COMPRESSiOn AlgorithmS}

The compression of DNA sequences is considered as one of the most challenging tasks in the field of data compression. In this connection the very first DNA compression and its subsequent algorithms BioCompress [10] and BioCompress-2 [11] detects exact repeats and complementary palindromes located in the sequence and Encode the factor by the size representation $(1, \mathrm{p})$ where 1 is the length of the factor and $\mathrm{p}$ is the position of its first occurrence. If the size is greater the factor then use two bit encoding. More memory references will require decoding the same, so the performance may degrade.

DnaPack [12] which uses hamming distance for the repeats and complementary palindromes and it is implemented by dynamic programming approach. So that it is not simple in design and it will require more time to execute and require more memory requirements also. The algorithm achieves a compression rate in an average of 1.6602 .

DNA compress [13] will work on approximation of repeats if number of tandem repeats more it saves bits to encode if not discard. Non repeated sequences will be appended to the sequence at the end. This algorithm achieves a compression rate only 1.72 bits per base. If there is no tandem repeat in the sequence it may run in worst case.

DNA Sequitur is a grammar based compression algorithm for DNA sequences which infers a context free grammar to represent input data..Designing a CFG for the given input data may leads to redundancies and constructing type2 language corresponding the grammar also leads to ambiguity.

The Lossless segment based compression enables part by part decompression by introducing non base character so that it will save memory requirements but it is applicable well on repeating sequences are more and more in the sequence. If such sequences like AT-rich DNA, which constitutes a distinct fraction of the cellular DNA of the archaebacterium Methanococcus voltae, consists of non-repetitive sequences, so part by part decompression is little bit tedious.

\section{RELATED EXISTING ALGORITHMS}

Compression methods are fall into two categories.

- Statistical methods which compress data by replacing the shorter code. Huffman code comes under this category and it's not suitable larger sequences.

- Dictionary based replacing larger strings by shorter code. Cfact which searches the longest exact matching repeat using tree data structures

Based on the above methods some loss less compression algorithms strived based on two bits encoding schemes i.e. $A(00), C(01), G(10)$, and $T(11)$. Huffbit compress [14], genbit compress [15] algorithms are explained performance analysis (Best, Avg and Worst) based on repetitive and non repetitive bases of DNA and computed results. Suppose in the given sequence more and more tandem repeats are there then [14], [15] will run in Beast case and achieves bountiful compression ratios, if not the same may run in worst case and achieves in an average 2.323 bits per bases [14], [15]. Our proposed algorithm PGBC(Partitioned group binary compression) techniques will achieves best compression ration even the given DNA sequence may doesn't contain tandem repeats or very less tandem repeats which can consider as minute in a very larger sequence like category of maze and rice grass sequences Our proposed algorithms are better suitable for non-repetitive DNA sequences in genome and which is achieving in an average of 1.333 bits per bases which is far better than all existing techniques.

\section{Proposed Algorithm}

Our proposed algorithms are developed based on the comparative study of existing techniques and we are starching on non-repetitiveness of DNA sequences. Existing techniques are run in worst case if any DNA sequences contain no tandem repeats. Here we are working with worst-case scenarios and achieves better compression rates in terms bits per symbols.

\section{A. Idea behind the Algorithm}

Every DNA sequence contain $\{A, C, G, T\}$ nucleotides where each literal is named as BASE and encoded in two bits as follows

$$
A=00, C=01, G=10 \text { and } T=11 \text {. }
$$

Compression ratio is calculated encoded bits per Bases. Compression Ratio = Encoded Bits/Bases.

\section{B. Plan of Work}

Here we took and input sequence as sample DNA (which doesn't contain any tandem repeats) of length $\mathrm{n}$ and divides it into $\mathrm{n} / 4$ fragments (where each fragment contain four bases i.e. $A, C, G$ and $T$ ). In Encoding process every six fragments can grouped as partition $(P)$ which contains two sub partitions $\left(F_{h}\right.$ and $S_{h}$ ). We can substitute its equivalent binary bits before making sub partitions and later we can group it into single main partition set. $\left(G_{\mathrm{s}}\right)$. In decoding process we can do the reverse or encoding to retain loss less DNA property. Finally we will calculate will group all the partition the equations are total number of encoded bits by grouping all the part ions.

Suppose if we took sample sequence of DNA which contain 72 bases then by applying PGBC techniques it will fragmented into $\mathrm{n} / 4$ i.e. 18 fragments, 3 partitions which will contain 6 sub partitions and then grouped it into single main partition. This will represent number of encoded bits in the given sequence. Our PGBC technique work as follows

$$
\begin{gathered}
N=\text { length of the given sequance } \\
F h=\text { First half sub partition } \\
\text { Sh }=\text { Second half sub partiotion } \\
\text { Ps = Partition set } \\
\text { Gs }=\text { Group partition set } \\
E g b=\text { Encoded group bits } \\
C r=\text { Compression ratio }
\end{gathered}
$$

Partition set $\left(P_{s)}\right.$ can calculate as follows. 


$$
P s=F h+S h
$$

Group partition set can calculate as follows

$$
G s=\sum_{s=0}^{\mathrm{m}}(P S)
$$

Here $G_{s}$ will represent the binary equivalent numeric (nearest to integer) in terms of Bytes storage. (Suppose if we will implement the technique in $C$ language unsigned int will require 4 bytes of storage).Here $m=n$ i.e. length of the given sequence.

Total Number of Encoded group bits are calculated as follows.

$$
E g b=\sum_{s=0}^{\mathrm{n}}(G s)
$$

Finally compression Ratio can calculate as follows.

$$
C r=E g b / N
$$

\section{Analysis}

Length of the given sequence $N=72$ Bases.

Then possibly 3 part ion sets which will include 6 sub partitions (3-Fh and 3-Sh) and finally we can group all partitions into a Group partition set.

$$
P s=P 1+P 2+P 3 \text {. }
$$

We will calculate binary equivalent numeric(which is nearer to integer) value for each partition in terms of Bytes storage(suppose if we will store each partition in $\mathrm{C}$ we will require two bytes if we can accommodate on int if not we can go for unsigned long).

$$
G_{s}=P_{s(p 1+p 2+p 3)}
$$

Total Number of Encoded Bits

$$
E_{g b}=G_{s}=P_{s}
$$

Every partition may contain 24 bases so it may not fit in integer so that we can store in unsigned long. So totally our sequence is divided into three partitions and grouped as one set . So totally we require 12 bytes to store.

$$
\begin{gathered}
E g b=P 1+P 2+P 3 \\
=4+4+4=12 \text { Bytes }(96 \text { bits })
\end{gathered}
$$

Finally we calculate Compression Ratio as follows.

$$
\begin{aligned}
C r & =E g b / N \\
& =96 / 72 \\
& =1.333 \text { (bits per Bases.) }
\end{aligned}
$$

Encoding and decoding algorithms for DNA compression is as follows.

\section{Encoding Algorithm}

INP: input String

OPS: Encoded String

\section{Procedure Encode}

Begin

- Group INS into equivalent fragments as four bases

- Generate all possible combinations of DNA and it will contain non- repetitive (our INS assumed as no tandem repeats).

- Group six fragments into partition set which will consist of two subpartions.

- Assign binary bits(0\&1) for every base of DNA like

$$
A=00, C=01, G=10 \text { and } T=11
$$

- Calculate Gs for every Ps in INP till eof INP

- Calculate Egb for every Gs till eof INP

- Repeat the steps 4 and 5 until the length of the INP

- Transfer the sequence Egb to the output string i.e. OPS String.

End.

\section{E. Decoding Algorithm}

INP: input String

OPS: Decoded String

\section{Procedure Decode}

Begin

- Generate all possible combinations of $(A, C, G, T)$

- Read the binary data of each sub partition from OPS and assign the two bits by equivalent Base s $(00=A, 01=C$, $10=G$ and $11=T$ ) and then store it in an array till eof

- Repeat step 2 until eof INS is reached and calculate Dgb and Ds in the reverse process.

- Transfer the sequence Db to the input String i.e. INP End.

\section{EXAMPLE AND COMPARISON}

Let us consider the sequence.

Sequence1:

ACGT GCGC GATC GCCT GCTA GGCG TACG TCGC AGGC GATC GATG TGCT AGAT CAGA TGAC TCAG TGCA CGAT.

Sequence length $($ no of bases $)=72$.

Bytes required to store in a text file $=72$ Bytes.

The above sequence doesn't contain tandem repeats so existing algorithms like Huffbit compress,Genbit Compress and Dnabit compress may run on worst case and require more bits to encode the sequence.

Huffbit,GenBit and Dna compress =162 bits(2.25)

Genbit Compress (Tool based) $=160$ bits $(2.23)$

PGBC Technique $($ Compression $)=96$ bits $(1.333)$

\section{CONCLUSION AND FUTURE WORK}

By using of our algorithm we can encode every base by 1.33 bits. By applying of ours we are saving nearer of 8 bytes to encode the given sequence, compression may vary with 
size of the sequence. So our technique is far better than existing ones and we can apply this technique on non repetitive DNA sequences of genomes. If the given sequence can contain tandem repeats also our technique will achieve same compression rate in an average. In addition to that existing techniques uses dynamic programming to compress the sequence which is complex in implementation and time consuming. Our technique is implemented without dynamic programming approach, so it is simple and fast. The simplicity of this will reduce the complexity in processing and definitely it will be the invaluable tool in Bio informatics era. Our algorithm can be extended to any tool based approach.

\section{ACKNOWLEDGMENT}

We would like thank other members of the Bio -Informatics teams (Faculty of CSE and IT) at Sphoorthy Engineering college Nadergul(V),R.R Dist,Hyderabad. I am very much greatful to my mother and father V.subba lakshmamma and V.C Obanna in every patho of my success.Last but not least students of CSE in sphoorthy for sharing their ideas with us in refining of our architecture

\section{REFERENCES}

[1] E. Schrodinger, Probability relations between separated systems, Cambridge, UK: Cambridge University Press, 1944.

[2] R. Giancarlo et al., A synopsis Bioinformatics, vol. 25, pp. 1575, 2009.

[3] E. V. Koonin, Bioinformatics, vol. 15, pp. 265, 1999.

[4] J. C. Wooley, J. Comput. Biol., vol. 6, pp. 459, 1999.
[5] C. H. Bennett et al., IEEE Trans.Inform.Theory, vol. 44, pp. 4, 1998.

[6] S. Grumbach and F Tahi, Journal of Information Processing and Management, vol. 30, no. 6, pp. 875, 1994.

[7] E Rivals et al., "A guaranteed compression scheme for repetitive DNA sequences,” LIFL, Lille I University, technical report IT-285, 1995.

[8] X. Chen et al., "A compression algorithm for DNA sequences and its applications in Genome comparison," in Proc. the Fourth Annual International Conference on Computational Molecular Biology, Tokyo, Japan, April 8-11, 2000.

[9] T. C. Bell et al., A Novel DNAZIP Tool for Zipping of Genome Sequences by Linear Bounded Data Structure, Newyork: Prentice Hall 1990.

[10] J. Ziv and A. Lempel, IEEE Trans. Inf. Theory, vol. 23, pp. 337, 1977.

[11] A. Grumbach and F. Tahi, in Proc. the IEEE Data.

[12] DNA compression is challenge is revisited Beshad Behajadi.

[13] A. AppaRao, "DNABIT compress-compression of DNA sequences," in Proc. the Bio medical Informatics, 2011.

[14] A. AppaRao, "HuffBit compress-compression of DNA using extended binary trees," in Proc. the Computational Biology and Bio Informatics, 2009.

[15] A. AppaRao, "Genbit compress-compression of DNA sequences," in Proc. the Computational Biology and Bio Informatics, 2011.

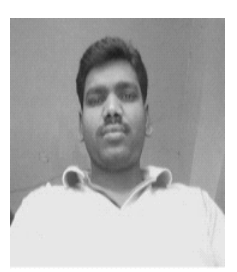

V. Hari Prasad is an Assoc. professor, who obtained B.Tech CSE from Jntu University, Anantapur, an M.Tech CSE from Jntuceh, Hyd. He is pursuing research in the area of Bio Informatics at Jntu Kakinada, A.P as a External Research scholar in CSE $\mathrm{He}$ has 10 years of teaching experience in various Engineerig colleges. Presently, he is heading the CSE Dept at Sphoorthy Engineering college, Nadergul(V), Hyd. He is a Life Member of MISTE and Member of

IEEE and UGC-NET qualified. He presented papers at International \& National conferences on various domains. His interested areas are Bio Informatics, Databases, and Artificial Intelligence. 